\title{
Antioxidant Supplement Improves the Pregnancy Rate in Patients Undergoing in Vitro Fertilization for Unexplained Infertility
}

\author{
Ayse Z. Ozdemir¹, Bulent Ayas', Davut Guven¹, Abdulkadir Bakay², Pervin Karlı ${ }^{*}$ \\ ${ }^{1}$ Ondokuz Mayıs University IVF Center, Ondokuz Mayıs University Hospital, Samsun, Turkey \\ ${ }^{2}$ Department of Obstetrics and Gynaecology, Ondokuz Mayıs University Hospital, Samsun, Turkey \\ ${ }^{3}$ Amasya University, Department of Obstetrics and Gynecology, Amasya, Turkey \\ Email: aysezehra.ozdemir@hotmail.com, ayasb@omu.edu.tr, davutguven55@gmail.com,drkadirbakay@gmail.com, \\ *parpi2300@hotmail.com
}

How to cite this paper: Ozdemir, A.Z. Ayas, B., Guven, D., Bakay, A. and Karlı, P. (2019) Antioxidant Supplement Improves the Pregnancy Rate in Patients Undergoing in Vitro Fertilization for Unexplained Infertility. Open Journal of Obstetrics and Gynecology, 9, 1-9.

https://doi.org/10.4236/ojog.2019.91001

Received: December 5, 2018

Accepted: January 1, 2019

Published: January 4, 2019

Copyright (c) 2019 by authors and Scientific Research Publishing Inc. This work is licensed under the Creative Commons Attribution International License (CC BY 4.0).

http://creativecommons.org/licenses/by/4.0/

\section{(c) (i) Open Access}

\begin{abstract}
Objective: This is a retrospective study to evaluate the effect of using the antioxidants containing coenzyme Q10 (CoQ10) and omega-3 in patients undergoing in vitro fertilization and intracytoplasmic sperm injection (IVF-ICSI) for unexplained infertility. Materials Methods: A total of 299 patients undergoing IVF-ICSI for unexplained infertility at the IVF center of Ondokuz Mayıs University were studied. The subject group consisted of 135 patients who used OMEPA Q10 (Co Q10 $100 \mathrm{mg}$, omega-3 $600 \mathrm{mg}$, eicosapentaenoic acid $300 \mathrm{mg}$, docosahexaenoic acid $230 \mathrm{mg}$ ) for two months before ovarian stimulation. A group of 164 patients who did not use the supplement were evaluated as the control group. Results: The pregnancy rate of patients using antioxidants was statistically higher than that of those who did not use the supplement $(\mathrm{p}<0.05)$. The pregnancy rates of patients in the two groups were $32.9 \%$ and $49.6 \%$ respectively. The pregnancy rate of patients using antioxidants was statistically higher than that of those who did not use the supplement $(\mathrm{p}<0.05)$ seemed not enough. Conclusion: We concluded that using antioxidants that contain Co Q10 and omega-3 in patients undergoing IVF-ICSI for unexplained infertility increased the clinical pregnancy rate. We recommend using antioxidants that contain Co Q10 and omega-3 in infertile patients.
\end{abstract}

\section{Keywords}

CoQ10, Omega-3, Antioxidant, Unexplained Infertility, In Vitro Fertilization 


\section{Introduction}

Today, despite many advances in infertility treatment, the rate of achieving live pregnancy after the first cycle of in vitro fertilization (IVF) is still around 29\% [1]. Therefore, various alternative treatments are used to increase IVF success. Nutritional supplements are one of these alternative treatments. Antioxidants are the most important nutritional supports; vitamins $\mathrm{C}$ and $\mathrm{E}$, omega-3, polyunsaturated fatty acids, melatonin, L-arginine, and coenzyme Q10 (Co Q10) are the main antioxidants used [2]. Many in vivo and in vitro free oxygen radicals occur during assisted reproduction processes [3]. These radicals prevent oocyte maturation [4] and embryo growth [5]. The use of antioxidants can contribute to the IVF process by eliminating these oxygen radicals. In unexplained infertility patients, it has been found that free oxygen radicals increased and some antioxidants decreased in follicular fluid [6]. Co Q10, also referred to as ubiquinone, is found in the inner membrane of mitochondria [7] and helps mitochondrial energy production by providing electron transport [8]. This energy production is vital for the continuation of normal reproductive function, since oocyte maturation, fertilization, and implantation of the embryo into endometrium are energy-dependent processes [9] [10]. Unlike other cells, oocytes and early embryos completely derive their energy requirement from oxidative phosphorylation [11]. Omega-3 consists of alpha-Linoleic acid (ALA), eicosapentaenoic acid (EPA) and docosahexaenoic acid (DHA). Omega-3 is essential for the synthesis of cholesterol, the precursor of estradiol synthesis. Studies have shown that omega-3 uptake contributes positively to embryo morphology [7]. Unexplained infertility diagnoses include patients for whom standard tests cannot identify the cause of infertility. Such patients constitute $15 \%-30 \%$ of the infertile patient population [12] [13]. The inability to detect causes of infertility with known diagnostic methods supports the idea that molecular-level defects or nutrition problems are the main cause of infertility. A lack of several micronutrients was detected in patients with unexplained infertility [14]. This deficiency may decrease the quality of oocytes by causing an increase of oxygen radicals in oocyte microenvironments [15]. Thus, it has been suggested that antioxidant supplements may improve the success of IVF by providing a positive effect on oocyte quality. This study investigates this suggested effect of omega-3 and Co Q10 usage in order to determine whether it can improve IVF success in unexplained infertility patients.

\section{Materials and Methods}

This study was carried out on 299 patients with unexplained infertility between 21 and 40 years of age; all were enrolled at the IVF center of Ondokuz Mayis University between January 2008 and December 2017. The unexplained infertility diagnoses were made according to the Practice Committee of the American Society for Reproductive Medicine (ASRM). Spermiogram and hysterosalpingography (HSG) were requested from each couple. Early follicular phase follicle 
stimulating hormone (FSH) value was measured and antral follicle count was performed by vaginal ultrasonography. This study included men with normal spermiogram analysis according to WHO2010 [16] criteria and ovulatory women below 40 years of age, no pathology on HSG and FSH value $<15$. At least two cycles of controlled ovarian hyperstimulation and intrauterine insemination (COH-IUI) were performed on patients before IVF-ICSI. Patients with polycystic ovary syndrome were excluded from the study. One hundred and thirty five patients used OMEPA Q10 (Co Q10100 mg, omega-3 $600 \mathrm{mg}$, EPA $300 \mathrm{mg}$, DHA $230 \mathrm{mg}$; TAB, Turkey) for two months before ovarian stimulation. These were registered as the subject group, while 164 patients who did not take the supplement were evaluated as the control group. As all patients recorded to the center after 2013 used omega 3 and Co Q10 as antioxidant routinely so they were involved in the subject group, patients before the date were included in the control group. All patients were examined during the early follicular phase. Recombinant FSH (Gonal F 450 IU; Merck Serono, Spain) and human menopausal gonadotropin (HMG) (Merional 150 IU; Ali Raif, Turkey) were subcutaneously used for ovarian stimulation. Ovarian stimulation was performed with the antagonist protocol. An appropriate gonadotropin dose was administered based onantral follicle count, FSH level, patient age, and body mass index. During stimulation, FSH, LH, E2, progesterone and serial ultrasonography measurements were made. When a follicle with a diameter of $12 \mathrm{~mm}$ or more was detected, the antagonist was given until the day of oocyte pick up (OPU). When more than two follicles $17 \mathrm{~mm}$ or larger in diameter appeared in serial ultrasonography measurements, ovulation was triggered by giving Human chorionic gonadotropin (hCG) subcutaneously (Ovitrelle $250 \mathrm{mcg}$; Merck Serono, Switzerland). OPU was scheduled for 36 hours after hCG injection. Each patient received $100 \mathrm{mg}$ of progesterone intramuscularly (Progest an $50 \mathrm{mg}$; Kocak, Turkey) and $8 \mathrm{mg}$ of estradiol orally (Estrofem $2 \mathrm{mg}$; Novo Nordisk, Denmark) for luteal support. We mentioned the only day 5 embryo transfers for this study. Considering patient age and embryo quality and number, either one or two embryos were transferred. Clinical pregnancy was defined by the appearance of the gestational sac on ultrasonography. The study protocol was approved by the Ethical Committee of the Medical Faculty of OndokuzMayıs University (OMU-KAEK 2018/16).

\section{Statistical Analysis}

The data were analyzed with IBM SPSS V.23. The appropriateness of the normal distribution of the data was examined by the Shapiro-Wilk test. While comparing non-normal distribution data according to antioxidant usage, a non-parametric method, the Mann-Whitney $U$ test, was used. When the categorical data were analyzed, the chi-square test was used. Quantitative data were presented in the form of a median (min-max), while the qualitative data were presented as a frequency (percent). The significance level was taken as $\mathrm{p}<0.05$. 
Power analysis results estimated that it would be sufficient to take 60 cases in the patient group and 120 cases in the control group to have $95 \%$ confidence and $81.3 \%$ test power (Gat et al., 2016).

\section{Results}

Two hundred ninety-nine patients were included in the study. While 164 did not use any antioxidants, 135 patients used the supplement. There is not a meaningful difference between the groups using and not using antioxidants according to age, antral follicle count or basal FSH ( $\mathrm{p}>0.05$ ) (Table 1). The median value of gonadotropin doses was found to be 2550 IU in the patients who used antioxidants and $3600 \mathrm{IU}$ in patients who did not use antioxidants; the difference between the groups was statistically significant $(\mathrm{p}<0.01)$. There is no significant difference between the groups regarding other parameters $(p>0.05)$ (Table 2). The pregnancy rate of the patients using antioxidants was statistically higher than those who did not use the supplement $(\mathrm{p}<0.05)$; the rate of pregnancy for the antioxidant group was $49.6 \%$, and the rate for the other group was $32.9 \%$ (Table 3).

\section{Discussion}

The results of this study indicated that clinical pregnancy rate significantly increased when omega-3 and Co Q10 were used as an antioxidant treatment for patients with unexplained infertility undergoing IVF-ICSI. In addition, the total gonadotropin dose required for stimulation was significantly lower in patients using antioxidants. The information available in the literature supports these findings. It has been shown that increased oxidative stress is a reason for infertility [17]. Oxidative stress is the result of antioxidant mechanisms being inadequate in clearing increased free oxygen radicals. Free oxygen radicals occur in vivo in both the male and female genital tract and in vitro during IVF procedures [3]. While certain levels of oxygen radicals are required for normal reproductive function, increasing levels of the radicals damage reproductive cells [18]. Increased free oxygen radicals disrupt nearly all components of the cell proteins, lipids, nucleic acids, carbohydrate structures and so on this damages the mitochondrial function and therefore reduces energy production. This negatively affects oocyte maturation, which is an energy-dependent process, and therefore

Table 1. Prestimulation assessment.

\begin{tabular}{cccc}
\hline & $\begin{array}{c}\text { Non-antioxidant group } \\
(\mathrm{n}=164)\end{array}$ & $\begin{array}{c}\text { Antioxidant group } \\
(\mathrm{n}=135)\end{array}$ & $\mathrm{P}$ \\
\hline Age & $31(21-39)$ & $32(22-39)$ & 0.058 \\
Number of antral follicles & $5(5-28)$ & $6(5-16)$ & 0.053 \\
Basal FSH (mIU/ml) & $10(2-15)$ & $12(3-15)$ & 0.055 \\
BMI & 24.3 & 25.9 & 0.57 \\
\hline
\end{tabular}

Data expressed as median (min-max). 
Table 2. Stimulation outcomes for both groups.

\begin{tabular}{cccc}
\hline & $\begin{array}{c}\text { Non-antioxidant group } \\
(\mathbf{n}=\mathbf{1 6 4})\end{array}$ & $\begin{array}{c}\text { Antioxidant group } \\
(\mathbf{n}=135)\end{array}$ & $\mathbf{P}$ \\
\hline $\begin{array}{c}\text { Estradiol level at hCG } \\
\text { day (pg/ml) }\end{array}$ & 1500 & 1400 & 0.407 \\
$\begin{array}{c}\text { Total amount of } \\
\text { gonadotropins } \\
\text { dose (IU/ml) }\end{array}$ & $(184-5400)$ & $(434-6000)$ & $<0.001$ \\
$\begin{array}{c}\text { Number of oocytes } \\
\text { collected }\end{array}$ & 3600 & 2550 & 0.960 \\
$\begin{array}{c}\text { Metaphase II oocytes } \\
\text { Number of oocytes } \\
\text { fertilized }\end{array}$ & $8(0-29)$ & $(1000-6300)$ & 0.855 \\
\hline
\end{tabular}

Data expressed as median (min-max).

Table 3. Pregnancy rates.

\begin{tabular}{cccc}
\hline & Non-Antioxidant group & Antioxidant group & P \\
\hline $\begin{array}{c}\text { Number of pregnancy-free } \\
\text { patients (\%) }\end{array}$ & $110(67.1)$ & $68(50.4)$ & \\
$\begin{array}{c}\text { Number of clinical pregnancies } \\
(\%)\end{array}$ & $54(32.9)$ & $67(49.6)$ & 0.002 \\
\hline
\end{tabular}

affects oocyte fertilization. Turi et al. showed that the Co Q10-protein value in mature oocytes is higher than in dysmorphic oocytes [19]. Ben-Meir et al. have shown that when the process of Co Q10 synthesis is damaged in rats, energy production is reduced, leading to infertility caused bymeiotic spindle anomalies [9]. Levels of glutathione peroxidase enzyme, an antioxidant, were found to be low in the follicular fluid of unexplained infertility patients [6]. It is estimated that in these individuals, Co Q10 taken in the diet may clear increased oxygen radicals in the follicular microenvironment and increase energy production leading to a better oocyte formation. Gat et al. did not find a statistically significant increase in pregnancy rates between poor ovarian reserve patients using Co Q10 in combination with dehydroepiandrosterone sulfate (DHEA) and those using DHEA alone [20]. However, a control group was not used in this study; DHEA was routinely given to both groups. On the other hand, Bentov et al. showed that use of Co Q10 increased pregnancy rates in patients who underwent IVF-ICSI in randomized controlled trials, but this result was not statistically significant because of the low number of patients [8]. This finding is in agreement with the results of our study. Youssef et al. used antioxidant preparations containing vitamins $\mathrm{A}, \mathrm{C}$, and $\mathrm{E}$, zinc, molybdenum, selenium, biotin, and bioflavonoids in unexplained infertility groups; these groups showed no difference in clinical pregnancy rates [21]. Despite the similar patient group, the significant increase in clinical pregnancy rates revealed in the current study may be due to the fact that CoQ10 is not only an antioxidant but also an agent that increases mitochondrial energy production. Besides increasing the production of mito- 
chondrial energy by providing electron transport, Co Q10 saves the mitochondria from oxidative stress [8]. Considering that oocytes and embryos receive their energy needs from oxidative phosphorylation, the presence of adequate and necessary energy levels seems essential for quality oocyte and embryo development. Studies have implicated that chromosomal disorders, the arrest of oocyte maturation, and impaired embryo development occur because of failures in the mechanism of oxidative phosphorylation [9] [10] [22]. The study performed by Akarsu et al., which measured Co Q10 levels in follicular fluid from patients undergoing IVF-ICSI for unexplained infertility, put forth that higher quality oocytes and embryos and a higher pregnancy rate were found in patients with high Co Q10 levels [23]. The high efficiency of Co Q10 as a lipid soluble antioxidant is proven by its localization, effective reactivation, and relatively high concentration [24]. In the present study, the use of antioxidants reduced the total gonadotropin dose administered for ovarian stimulation although there is no change in stimulation protocols. Similar findings were observed by Gat et al. who found that Co Q10 reduced the total gonadotropin dose in patients with poor ovarian reserve [20]. The authors argued that Co Q10 could increase the sensitivity of follicles to gonadotropins. Turi et al. showed the presence of Co Q10 in the follicular fluid first, and they speculated that it may have distinct roles from that found in the mitochondrial inner membrane [19]. This result supports the findings of the present study; particularly in confirming that Co Q10 may enhance the sensitivity of follicles to gonadotropins. Endometrial secretions and fluid have been shown to be effective in feeding the embryo during the preimplantation phase [25]. Increased pregnancy rates may also be associated with the elimination of oxidants and energy deficiencies in the endometrial fluid. It was also found that the implantation potential of the embryo is related to the energy content of the embryo [10]. Although this study is retrospective, it may be a guide for prospective studies to be performed in this area, since it includes a large population of patients. The most important limitation of our study is that omega-3 and Co Q10 are used together; therefore, it is not possible to comment on which is more effective or whether they have a synergistic effect in increasing the pregnancy rate. This study is an observation of an apparent change in clinical pregnancy that coincided with the introduction of a nutritional supplement. The interpretation of this needs to be much more cautious as there are many other factors that could potentially account for this improvement i.e. laboratory experience although we didn't have any change in protocols for unexplained infertility patients during the time.

\section{Conclusion}

We found that using antioxidants containing Co Q10 and omega-3 in patients with unexplained infertility undergoing IVF-ICSI increased the clinical pregnancy rate. There is a need for randomized controlled studies in order to precisely show the effect of CoQ10 and omega-3 when used in the treatment of unexplained infertility. 


\section{Funding Statement}

This research received no specific grant from any funding agency in the public, commercial, or not-for-profit sectors.

\section{Conflicts of Interest}

The authors declare no conflicts of interest regarding the publication of this paper.

\section{References}

[1] McLernon, D.J., Maheshwari, A., Lee, A.J. and Bhattacharya, S. (2016) Cumulative Live Birth Rates after One or More Complete Cycles of IVF: A Population-Based Study of Linked Cycle Data from 178,898 Women. Human Reproduction, 31, 572-581. https://doi.org/10.1093/humrep/dev336

[2] Showell, M.G., Brown, J., Clarke, J. and Hart, R.J. (2013) Antioxidants for Female Subfertility. In: Showell, M.G., Ed., Cochrane Database of Systematic Reviews (Vol. 5), John Wiley \& Sons, Ltd., Chichester, UK. https://doi.org/10.1002/14651858.CD007807.pub2

[3] Rakhit, M., Gokul, S.R., Agarwal, A. and du Plessis, S.S. (2013) Antioxidant Strategies to Overcome OS in IVF-Embryo Transfer. In: Studies on Women's Health, Humana Press, Totowa, NJ, 237-262.

[4] Agarwal, A., Gupta, S., Abdel-Razek, H., Krajcir, N. and Athayde, K. (2006) Impact of Oxidative Stress on Gametes and Embryos in an ART Laboratory. The Clinical Embryologist, 9, 5-22.

[5] Caamaño, J.N., Ryoo, Z.Y., Thomas, J.A. and Youngs, C.R. (1996) Beta-Mercaptoethanol Enhances Blastocyst Formation Rate of Bovine in $\mathrm{Vi}$ tro-Matured/in Vitro-Fertilized Embryos. Biology of Reproduction, 55, 1179-1184. https://doi.org/10.1095/biolreprod55.5.1179

[6] Paszkowski, T. and Clarke, R.N. (1996) Antioxidative Capacity of Preimplantation Embryo Culture Medium Declines Following the Incubation of Poor Quality Embryos. Human Reproduction, 11, 2493-2495. https://doi.org/10.1093/oxfordjournals.humrep.a019146

[7] Balercia, G., Mancini, A., Tirabassi, G. and Pontecorvi, A. (2017) Coenzyme Q10 in Male Infertility. In: Antioxidants in Andrology, Springer International Publishing, Cham., 43-57. https://doi.org/10.1007/978-3-319-41749-3

[8] Bentov Y, Hannam, T., Juriscova, A., Esfandiari, N. and Casper, R. (2014) Coenzyme Q10 Supplementation and Oocyte Aneuploidy in Women Undergoing IVF-ICSI Treatment. Clinical Medicine Insights: Reproductive Health, 8, CMRH.S14681. https://doi.org/10.4137/CMRH.S14681

[9] Ben-Meir, A., Burstein, E., Borrego-Alvarez, A., Chong, J., Wong, E., Yavorska, T., Jurisicova, A., et al. (2015) Coenzyme Q10 Restores Oocyte Mitochondrial Function and Fertility during Reproductive Aging. Aging Cell, 14, 887-895. https://doi.org/10.1111/acel.12368

[10] Van Blerkom, J., Davis, P. and Lee, J. (1995) ATP Content of Human Oocytes and Developmental Potential and Outcome after in-Vitro Fertilization and Embryo Transfer. Human Reproduction, 10, 415-424. https://doi.org/10.1093/oxfordjournals.humrep.a135954

[11] Hammiche, F., Vujkovic, M., Wijburg, W., Vries, J.H.M. De, Macklon, N.S., Laven, 
J.S.E., Kingdom, U., et al. (2011) Increased Preconception Omega-3 Polyunsaturated Fatty Acid Intake Improves Embryo Morphology. Fertility and Sterility, 95, 1820-1823. https://doi.org/10.1016/j.fertnstert.2010.11.021

[12] Practice Committee of the American Society for Reproductive Medicine (2006) Optimal Evaluation of the Infertile Female. Fertility and Sterility, 86, 264-267.

[13] Practice Committee of the American Society for Reproductive Medicine (2006) Effectiveness and Treatment for Unexplained Infertility. Fertility and Sterility, 86, 111-114. https://doi.org/10.1016/j.fertnstert.2006.07.1475

[14] Noventa, M., Quaranta, M., Vitagliano, A., Cinthya, V., Valentini, R., Campagnaro, T., Gizzo, S., et al. (2015) May Underdiagnosed Nutrition Imbalances Be Responsible for a Portion of So-Called Unexplained Infertility? From Diagnosis to Potential Treatment Options. Reproductive Sciences, 23, 812-822. https://doi.org/10.1177/1933719115620496

[15] Luddi, A., Capaldo, A., Focarelli, R., Gori, M., Morgante, G., Piomboni, P. and De Leo, V. (2016) Antioxidants Reduce Oxidative Stress in Follicular Fluid of Aged Women Undergoing IVF. Reproductive Biology and Endocrinology, 14, 1-7. https://doi.org/10.1186/s12958-016-0184-7

[16] Ford, W.C.L. (2010) Comments on the Release of the 5th Edition of the WHO Laboratory Manual for the Examination and Processing of Human Semen. Asian Journal of Andrology, 12, 59-63. https://doi.org/10.1038/aja.2008.57

[17] Gupta, S., Ghulmiyyah, J., Sharma, R., Halabi, J. and Agarwal, A. (2014) Power of Proteomics in Linking Oxidative Stress and Female Infertility. BioMed Research International, 2014, Article ID: 916212. https://doi.org/10.1155/2014/916212

[18] Sikka, S., Rajasekaran, M. and Hellstrom, W.J. (1995) Role of Oxidative Stress and Antioxidants in Male Infertility. Journal of Andrology, 16, 464-468. https://doi.org/10.1002/j.1939-4640.1995.tb00566.x

[19] Turi, A., Giannubilo, S.R., Brugè, F., Principi, F., Battistoni, S., Santoni, F. and Tiano, L. (2012) Coenzyme Q10 Content in Follicular Fluid and Its Relationship with Oocyte Fertilization and Embryo Grading. Archives of Gynecology and Obstetrics, 285, 1173-1176. https://doi.org/10.1007/s00404-011-2169-2

[20] Gat, I., Blanco Mejia, S., Balakier, H., Librach, C.L., Claessens, A. and Ryan, E.A.J. (2016) The Use of Coenzyme Q10 and DHEA During IUI and IVF Cycles in Patients with Decreased Ovarian Reserve. Gynecological Endocrinology, 32, 534-537. https://doi.org/10.3109/09513590.2015.1137095

[21] Youssef, M.A., Abdelmoty, H.I., Elashmwi, H.A., Abduljawad, E.M., Elghamary, N., Magdy, A. and Ali, A.M.S. (2015) Oral Antioxidants Supplementation for Women with Unexplained Infertility Undergoing ICSI/IVF: Randomized Controlled Trial. Human Fertility, 18, 38-42. https://doi.org/10.3109/14647273.2014.927595

[22] Takeuchi, T., Neri, Q.V., Katagiri, Y., Rosenwaks, Z. and Palermo, G.D. (2005) Effect of Treating Induced Mitochondrial Damage on Embryonic Development and Epigenesis. Biology of Reproduction, 72, 584-592.

https://doi.org/10.1095/biolreprod.104.032391

[23] Akarsu, S., Gode, F., Isik, A.Z., Dikmen, Z.G. and Tekindal, M.A. (2017) The Association between Coenzyme Q10 Concentrations in Follicular Fluid with Embryo Morphokinetics and Pregnancy Rate in Assisted Reproductive Techniques. Journal of Assisted Reproduction and Genetics, 34, 599-605. https://doi.org/10.1007/s10815-017-0882-x

[24] Bentinger, M., Brismar, K. and Dallner, G. (2007) The Antioxidant Role of Coenzyme Q. Mitochondrion, 7, 41-50. https://doi.org/10.1016/j.mito.2007.02.006 
[25] Leese, H.J., Hugentobler, S.A., Gray, S.M. and Morris, D.G., Sturmey, R.G., Whitear, S.-L. and Sreenan, J.M. (2008) Female Reproductive Tract Fluids: Composition, Mechanism of Formation and Potential Role in the Developmental Origins of Health and Disease. Reproduction, Fertility and Development, 20, 1-8.

https://doi.org/10.1071/RD07153 